\title{
Discrete-Time 2-Order Sliding Mode Fault-Tolerant Tracking Control for Non-Gaussian Nonlinear Stochastic Distribution Control Systems with Missing Measurements
}

\author{
Lifan Li and Lina Yao \\ School of Electrical Engineering, Zhengzhou University, Zhengzhou, Henan 450001, China \\ Correspondence should be addressed to Lina Yao; michelle_lnxq@126.com
}

Received 27 January 2020; Revised 3 April 2020; Accepted 6 May 2020; Published 5 June 2020

Academic Editor: Saleh Mobayen

Copyright (c) 2020 Lifan Li and Lina Yao. This is an open access article distributed under the Creative Commons Attribution License, which permits unrestricted use, distribution, and reproduction in any medium, provided the original work is properly cited.

\begin{abstract}
In this paper, a method of fault diagnosis (FD) and fault-tolerant tracking control (FTTC) is investigated for non-Gaussian nonlinear stochastic distribution control (SDC) systems with missing measurements. The phenomenon of the randomly occurring missing measurements is described as a Bernoulli process. The missing measurements during transmission are compensated with the data successfully transmitted at the previous moment. The residual signal of the fault diagnosis observer is different from that of the general system. Using the integral of the error of the output probability density function (PDF) as the driving information, the system state and fault can be estimated by an adaptive fault diagnosis observer. Then, a novel fault-tolerant tracking controller is designed based on a discrete-time 2-order sliding mode to make the post-fault PDF still track the target. Two simulated examples are included to illustrate the effectiveness of the theoretical results.
\end{abstract}

\section{Introduction}

Stochastic control is an important research field in control science. At present, research on Gaussian stochastic system control has achieved a lot of theoretical and practical results [1-3]. However, the nonlinear characteristics of the actual industry control system and the random noise which obeys the non-Gaussian distribution may lead to the existence of non-Gaussian random variables in the system. On the other hand, with the rapid development of precision instruments and data processing technology, the information that can be measured and used for feedback control is no longer limited to the measured value of the output signal but also can be the output PDF or statistical information set. How to solve these problems effectively is a new challenge for stochastic control theory. Motivated by this, Professor Wang Hong proposed a new research direction of stochastic control theory with the output PDF as the research object, which is called stochastic distribution control (SDC) theory [4]. After nearly a decade of development, the non-Gaussian stochastic distribution control is gradually forming a relatively complete framework for modeling theory and control theory research, including filtering [5, 6], iterative learning control [7], and tracking control [8-10].

The non-Gaussian stochastic distribution control theory has a wide range of applications, such as the control of molecular weight distribution of polymers in chemical process $[11,12]$, the gray distribution control of papermaking process [13], and the distribution control of temperature field in combustion process [4]. The applications mentioned above are complex large-scale industrial processes. Once the fault happens in these systems, it may reduce the stability of the system and cause huge economic losses. With the increasing demand for system security, the research on fault diagnosis and fault-tolerant control methods for non-Gaussian stochastic distribution systems has attracted more and more attention [14-18]. In [15], an iterative learning fault diagnosis observer is designed, which can be used to estimate both the fast-varying fault and the slow-varying fault. The fault diagnosis algorithm based on 
unknown input observer and sliding mode fault-tolerant controller are proposed in [16] for non-Gaussian uncertain stochastic distribution control systems with PDF approximation error. A new operational fault-tolerant control algorithm is proposed for collaborative stochastic distribution control systems [18].

In practical engineering applications, due to the complexity of the environment and the reliability of communication, the missing measurements are inevitable. The influence of missing measurements on the control performance of the system cannot be neglected. The research on state estimation and fault diagnosis for the system with missing measurements has attracted increasing interest. A consistent parameter estimation algorithm is proposed to solve the parameter estimation problem of linear systems with missing measurements in [19]. In order to deal with the state estimation problem for power systems with missing measurements [20], a constrained optimization approach based on the extended Kalman filter is carried out. For a class of nonlinear systems with logarithmic quantization and missing measurements, the corresponding state and fault estimation problem is transmitted into the recursive filtering problem by augmenting the state variables [21]. Until now, the studies on fault diagnosis and fault-tolerant control for non-Gaussian SDC systems with missing measurements are rare to the best of our knowledge.

The sliding mode control is a nonlinear and robust control strategy. To solve the problem of the nonlinear relationship between DC-link voltage and the control input, a new supertwisting sliding mode control algorithm is implemented in [22]. In [23], the disturbance observer based on fixed-time supertwisting algorithm and the controller based on fixed-time high-order sliding mode are designed for high-order dynamic systems. The main disadvantage of sliding mode control is the chattering problem. 2-order sliding mode control can effectively suppress chattering and improve the control accuracy [24]. The selection of design parameters of a discrete-time neural second-order sliding mode controller is studied for unknown nonlinear systems in [25]. In [26], a novel discrete-time second-order sliding mode control for multivariable systems is developed.

Based on the aforementioned discussion, we make the first attempt to address the problem of fault diagnosis and fault-tolerant control for the non-Gaussian SDC system with missing measurements. The main contents and contributions of this paper can be summarized as follows:

(1) In the presence of the randomly occurring missing measurements, a Lipschitz nonlinear SDC model is established. The phenomenon of the randomly occurring missing measurements is described as a Bernoulli process. In order to eliminate the influence of missing measurements, the missing measurement in the transmission process is compensated by the data successfully transmitted at the previous moment.

(2) An adaptive observer is used to estimate the system state and fault simultaneously. Then, a new discretetime 2-order sliding mode fault-tolerant tracking controller is given to guarantee the reachability of the sliding surface and make the postfault output PDF still track the target.

(3) Compared with the first-order sliding mode faulttolerant control, a better fault-tolerant tracking performance can be obtained by the proposed faulttolerant controller in this paper.

The rest of this paper is organized as follows. Section 2 presents the problem description. Fault diagnosis method is given in Section 3. In Section 4, the design of fault-tolerant tracking controller based on a discrete 2-order sliding mode is presented. Section 5 gives the simulation results, which is followed by some concluding remarks in Section 6 .

\section{Model Description}

For the nonlinear SDC system, $y \in[a, b]$ is defined as the bound and continuous random output variable of the system and $u(k)$ is the control input. The probability $P(a \leq y \leq b)$ can be depicted by the output $\operatorname{PDF} \gamma(y, u(k))[7,27]$, which can be defined as follows:

$$
P(a \leq y \leq b)=\int_{a}^{b} \gamma(y, u(k)) \mathrm{d} y .
$$

The discrete-time output PDF model based on linear B-spline [4] can be represented as follows:

$$
\gamma(y, u(k))=\sum_{s=1}^{N} \omega_{s}(u(k)) \varphi_{s}(y),
$$

where $\gamma(y, u(k))$ is the output PDF, $\omega_{s}(u(k))(s=1,2, \ldots, N)$ are the corresponding dynamic weights associated with the control input, and $\varphi_{s}(y)(s=$ $1,2, \ldots, N)$ are the prespecified basis functions.

According to the condition that $\int_{a}^{b} \gamma(y, u(k)) \mathrm{d} y=1$, equation (2) can be further expressed as follows:

$$
\gamma(y, u(k))=\varphi_{0}(y) W(k)+\omega_{N}(k) \varphi_{N}(y),
$$

where $\varphi_{0}(y)=\left[\varphi_{1}(y) \ldots \varphi_{N-1}(y)\right], W(k)=\left[\omega_{1}(k) \ldots\right.$ $\left.\omega_{N-1}(k)\right]^{T}$.

Define $\phi^{T}=\left(\phi_{1}, \phi_{2}, \ldots, \phi_{N-1}\right) \in R^{N-1}, \phi_{i}=\int_{a}^{b} \varphi_{i}(y) \mathrm{d} y$; it can be obtained that

$$
\gamma(y, u(k))=\varphi(y) W(k)+\psi(y)
$$

where

$\left.(y) / \phi_{N}\right)$.

$$
\varphi(y)=\varphi_{0}(y)-\left(\varphi_{N}(y) / \phi_{N}\right) \phi^{T}, \psi(y)=\left(\varphi_{N}\right.
$$

Remark 1. The linear B-spline model is simple and intuitive, which is the most mature model at present. There are other basic functions for approximating the output of PDF. In the square root B-spline model, the relationship between the output PDF and the weight is nonlinear, which increases the design difficulty of the controller. For the radial basis function (RBF) model, if the center and width are not properly selected, it will lead to a large approximation error [27]. Therefore, the linear B-spline model is used to approximate the output PDF in this paper. 
In this paper, the following non-Gaussian nonlinear SDC system is considered as follows:

$$
\begin{aligned}
x(k+1) & =A x(k)+B u(k)+g(x(k))+E f(k), \\
W(k) & =D x(k), \\
\bar{\gamma}(y, u(k)) & =\lambda_{k} \gamma(y, u(k))+\left(1-\lambda_{k}\right) \gamma(y, u(k-1)),
\end{aligned}
$$

where $x(k) \in R^{n}$ is the state vector, $W(k) \in R^{m}$ is the weight vector, and $f(k)$ is the fault vector. $E f(k)$ is the additive fault item. $A, B, E, D$ are known system parameter matrices with appropriate dimensions. $\lambda(k)$ is the random variable obeying the Bernoulli distribution. $\lambda(k)$ takes a value 0 or 1 , and $\operatorname{Prob}\{\lambda(k)=1\}=E\{\lambda(k)\}=\bar{\lambda} \quad$ and $\operatorname{Prob}\{\lambda(k)=0\}=1-\bar{\lambda}$. When $\lambda(k)=1$, it means that the data are successfully transmitted. However, when $\lambda(k)=0$, it indicates that missing measurements have occurred, which can be compensated by the data successfully transmitted at the previous moment.

$\bar{\gamma}(y, u(k))$ can be further expressed as follows:

$$
\bar{\gamma}(y, u(k))=\varphi(y) \bar{W}(k)+\psi(y),
$$

where $\bar{W}(k)=\lambda(k) D x(k)+(1-\lambda(k)) \bar{W}(k-1)$.

Assumption 1. $g(x(t))$ is a nonlinear function, and the following Lipschitz condition is satisfied for any $x_{1}(t)$ and $x_{2}(t)$ :

$$
\left\{\begin{array}{l}
g(0)=0, \\
\left\|g\left(x_{1}(k)\right)-g\left(x_{2}(k)\right)\right\| \leq\left\|\gamma\left(x_{1}(k)-x_{2}(k)\right)\right\|,
\end{array}\right.
$$

where $\gamma$ is a known Lipschitz constant.

Remark 2. Assumption 1 is a common assumption since many nonlinear terms in practical systems satisfy the Lipschitz condition.

\section{Fault Diagnosis}

The fault diagnosis observer is designed to estimate the size of the fault so as to provide accurate fault estimation information for the design of fault-tolerant controller. Based on the input signal $\bar{\gamma}(y, u(k))$ received by the fault diagnosis observer, the fault diagnosis observer can be constructed as follows:

$$
\begin{aligned}
\widehat{x}(k+1) & =A \widehat{x}(k)+B u(k)+E \widehat{f}(k)+L \varepsilon(k)+g(\widehat{x}(k)), \\
\widehat{W}(k) & =D \widehat{x}(k), \\
\widehat{\gamma}(y, u(k)) & =\varphi(y) \widehat{W}(k)+\psi(y), \\
\widehat{f}(k+1) & =v_{1} \widehat{f}(k)+v_{2} \varepsilon(k), \\
\varepsilon(k) & =\int_{a}^{b} \chi(y)(\bar{\gamma}(y, u(k)))-\widehat{\gamma}(y, u(k)) \mathrm{d} y,
\end{aligned}
$$

where $\hat{x}(k)$ is the state vector of the fault diagnosis observer, $\widehat{W}(k)$ is the estimation of the weight vector, and $\widehat{\gamma}(y, u(k))$ is the estimation of the output PDF. $\varepsilon(k)$ is the residual and $L$ is the adaptive gain to be designed for this fault diagnosis observer. $v_{1}$ and $v_{2}$ are learning operators to be determined later. $\chi(y)$ is a prespecified adjustment factor and cannot be equal to 1 .

Denote the diagnosis error vector and the fault error as follows:

$$
\begin{gathered}
e(k)=x(k)-\widehat{x}(k), \\
\tilde{f}(k)=f(k)-\widehat{f}(k) .
\end{gathered}
$$

Define $\Delta W(k)=W(k)-\bar{W}(k)$; the residual signal can be calculated as follows:

$$
\begin{aligned}
\varepsilon(k) & =\int_{a}^{b} \chi(y)(\bar{\gamma}(y, u(k)))-\widehat{\gamma}(y, u(k)) \mathrm{d} y \\
& =\int_{a}^{b} \chi(y) \varphi(y)(\bar{V}(k)-\widehat{W}(k)) \mathrm{d} y \\
& =\int_{a}^{b} \chi(y) \varphi(y) d y(V(k)-\Delta W(k)-\widehat{W}(k)) \\
& =\Sigma D e(k)-\Sigma \Delta W(k),
\end{aligned}
$$

where $\Sigma=\int_{a}^{b} \chi(y) C(y) \mathrm{d} y$.

According to (5) and (8), the following observation error dynamic system can be obtained

$$
\begin{aligned}
e(k+1)= & (A-L \Sigma D) e(k)+E \tilde{f}(k)+g(x(k)) \\
& -g(\hat{x}(k))+L \Sigma \Delta W(k) \\
= & (A-L \Sigma D) e(k)+E \tilde{f}(k)+G(k)+L \Sigma \Delta W(k) \\
= & (A-L \Sigma D) e(k)+E \tilde{f}(k)+D_{1} w(k) \\
\tilde{f}(k+1)= & f(k+1)-v_{1} f(k)+v_{1} \tilde{f}(k) \\
& -v_{2} \Sigma D e(k)+v_{2} \Sigma \Delta W(k) \\
= & \Delta f(k)+v_{1} \tilde{f}(k)-v_{2} \Sigma D e(k)+v_{2} \Sigma \Delta W(k) \\
= & v_{1} \tilde{f}(k)-v_{2} \Sigma D e(k)+D_{2} w(k),
\end{aligned}
$$

where $\quad G(k)=g(x(k))-g(\hat{x}(k)), \Delta f(k)=f(k+1)$ $-\Gamma_{1} f(k), \quad D_{1}=\left[\begin{array}{lll}I & 0 & L \Sigma\end{array}\right], D_{2}=\left[\begin{array}{lll}0 & I & v_{2} \Sigma\end{array}\right]$, and $w(k)=$ $\left[\begin{array}{lll}G(k) & \Delta f(k) & \Delta W(k)\end{array}\right]^{T}$.

Theorem 1. For the parameter $\kappa>0$, it is supposed that there exist matrices $P=P^{T}>0, Q=Q^{T}>0, R_{1}, R_{2}$, and $R_{3}$ such that the LMI

$$
\Phi=\left[\begin{array}{cccc}
-P+\kappa I & 0 & A^{T} P-D^{T} \Sigma^{T} R_{1}^{T} & -D^{T} \Sigma^{T} R_{2}^{T} \\
* & -Q+\kappa I & E^{T} P & R_{3}^{T} \\
* & * & -P & 0 \\
* & * & * & -Q
\end{array}\right]<0,
$$

holds, where $L=P^{-1} R_{1}, v_{2}=Q^{-1} R_{2}$, and $v_{1}=Q^{-1} R_{3}$. Then, observation error dynamic system (11) is stable when fault occurs.

Proof. Define the following Lyapunov function: 


$$
\pi(k)=e^{T}(k) \operatorname{Pe}(k)+\tilde{f}^{T}(k) Q \tilde{f}(k) .
$$

Then, it can be obtained that

$$
\begin{aligned}
\Delta \pi= & \pi(k+1)-\pi(k) \\
= & e^{T}(k+1) P e(k+1)+\tilde{f}^{T}(k+1) Q \tilde{f}(k+1) \\
& -e^{T}(k) P e(k)-\tilde{f}^{T}(k) Q \tilde{f}(k) \\
= & \left.(A-L \Sigma D) e(k)+E \tilde{f}(k)+D_{1} w(k)\right)^{T} P\left((A-L \Sigma D) e(k)+E \tilde{f}(k)+D_{1} w(k)\right) \\
& +\left(v_{1} \tilde{f}(k)-v_{2} \Sigma D e(k)+D_{2} w(k)\right)^{T} Q\left(v_{1} \tilde{f}(k)-v_{2} \Sigma D e(k)+D_{2} w(k)\right)-e^{T}(k) P e(k)-\tilde{f}^{T}(k) Q \tilde{f}(k) \\
= & e^{T}(k)(A-L \Sigma D)^{T} P(A-L \Sigma D) e(k)+2 e^{T}(k)(A-L \Sigma D)^{T} P E \tilde{f}(k)+2 e^{T}(k)(A-L \Sigma D)^{T} P D_{1} w(k) \\
& +2 \tilde{f}^{T}(k) E^{T} P D_{1} w(k)+w^{T}(k) D_{1}^{T} P D_{1} w(k)+\tilde{f}^{T}(k) E^{T} P E \tilde{f}(k)+\tilde{f}^{T}(k) v_{1}^{T} Q v_{1} \tilde{f}(k) \\
& +e^{T}(k)\left(v_{2} \Sigma D\right)^{T} Q v_{2} \Sigma D e(k)+w^{T}(k) D_{2}^{T} Q D_{2} w(k)-2 e^{T}(k)\left(v_{2} \Sigma D\right)^{T} Q v_{1} \tilde{f}(k)+2 \tilde{f}^{T}(k) v_{1}^{T} Q D_{2} w(k) \\
& -2 e^{T}(k)\left(v_{2} \Sigma D\right)^{T} Q D_{2} w(k)-e^{T}(k) P e(k)-\tilde{f}^{T}(k) Q \tilde{f}(k) \\
= & \bar{e}^{T}(k) \Phi_{1} \bar{e}(k)+w^{T}(k) \Phi_{2} w(k)+2 \bar{e}^{T}(k) \Phi_{3} w(k),
\end{aligned}
$$

where

$$
\begin{aligned}
& \Phi_{1}=\left[\begin{array}{cc}
(A-L \Sigma D)^{T} P(A-L \Sigma D)+\left(v_{2} \Sigma D\right)^{T} Q v_{2} \Sigma D-P & (A-L \Sigma D)^{T} P E-\left(v_{2} \Sigma D\right)^{T} Q v_{1} \\
* & E^{T} P E+v_{1}^{T} Q v_{1}-Q
\end{array}\right], \\
& \Phi_{2}=D_{1}^{T} P D_{1}+D_{2}^{T} Q D_{2}, \\
& \Phi_{3}=\left[\begin{array}{c}
(A-L \Sigma D)^{T} P D_{1}-\left(v_{2} \Sigma D\right)^{T} Q D_{2} \\
E^{T} P D_{1}+v_{1}^{T} Q D_{2}
\end{array}\right] .
\end{aligned}
$$

By using the Schur complement formula, it can be obtained that $\Phi<0 \Rightarrow \Phi_{1}+\kappa I<0$. Thus, it can be seen that

$\Delta \pi<-\kappa\|\bar{e}(k)\|^{2}+\left\|\Phi_{2}\right\|\|w(k)\|^{2}+2\left\|\Phi_{3}\right\|\|\bar{e}(k)\|\|w(k)\|$.

It is shown that when the inequality

$$
\|\bar{e}(k)\| \geq \kappa^{-1}\left(\left\|\Phi_{3}\right\|\|w(k)\|+\sqrt{\left(\kappa^{-1}\left\|\Phi_{3}\right\|+\left\|\Phi_{2}\right\|\right)\|w(k)\|^{2}}\right),
$$

is satisfied, it can be obtained that $\Delta \pi<0$. Then, observation error system (11) is stable.

\section{Discrete-Time 2-Order Sliding Mode Fault- Tolerant Tracking Control}

A new 2-order discrete-time sliding mode fault-tolerant tracking strategy is implemented based on the estimated fault information, which can guarantee perfect tracking performance even when the system is subject to the actuator fault. Denote the weight error vector as $e_{w}(k)=D x(k)-W_{g}$, where $W_{g}$ is the desired weight vector and $\gamma_{g}(y, u(k))=\varphi(y) W_{g}+\psi(y)$, where $\gamma_{g}(y, u(k))$ is the desired output PDF.

The discrete-time sliding function is designed as follows:

$$
s(k)=s(k-1)+e_{w}(k) .
$$

Furthermore, the following 2-order sliding mode function is designed as [26]

$$
\rho(k)=G s(k)+G \theta s(k-1)
$$

where $0 \leq \theta \leq 1$ and $G$ will be designed later to ensure the nonsingularity of GDB.

From equations (5) and (19), $\rho(k+1)$ can be expressed as follows: 


$$
\begin{aligned}
\rho(k+1)= & G s(k+1)+G \theta s(k) \\
= & G s(k)+G e_{w}(k+1)+G \theta s(k) \\
= & G e_{w}(k+1)+G(1+\theta) s(k) \\
= & G D x(k+1)-G W_{g}(k+1)+G(1+\theta) s(k) \\
= & G D(A x(k)+B u(k)+g(x(k))+E f(k)) \\
& -G W_{g}(k+1)+G(1+\theta) s(k) .
\end{aligned}
$$

Let $\rho(k+1)=0$, and the following equivalent controller can be obtained:

$$
\begin{aligned}
u_{\mathrm{eq}}(k)= & (\mathrm{GDB})^{-1} G\left(W_{g}(k+1)-(1+\theta) s(k)-D(A x(k)\right. \\
& +g(x(k))+E f(k))) .
\end{aligned}
$$

A discontinuous term is applied to ensure the robustness of the sliding mode control law [25]:

$$
u_{\text {dis }}(k)=u_{\text {dis }}(k-1)+(\mathrm{GDB})^{-1} h(-\delta \operatorname{sgn}((\rho(k))-q \rho(k))) \text {, }
$$

where $\delta<0, q>0,1-q h>0$.

The overall control law can be represented as follows:

$$
u(k)=u_{\mathrm{eq}}(k)+u_{\mathrm{dis}}(k) .
$$

Theorem 2. The trajectory of the closed-loop system can be driven onto the sliding surface in finite time with control law (23).

Proof. It can be deduced that

$$
\begin{aligned}
e_{w}(k+1)= & D x(k+1)-W_{g}(k+1) \\
= & D\left(A x(k)+B\left(u_{\mathrm{eq}}(k)+u_{\mathrm{dis}}(k)\right)\right. \\
& +g(x(k))+E f(k))-W_{g}(k+1) \\
= & D B u_{\mathrm{dis}}(k)-(1+\theta) s(k) .
\end{aligned}
$$

According to (19) and (23), it can be obtained that

$$
\begin{aligned}
\rho(k+1) & =G s(k+1)+G \theta s(k) \\
& =G s(k)+G e_{w}(k+1)+G \theta s(k) \\
& =G e_{w}(k+1)+G(1+\theta) s(k) \\
& =G D B u_{\text {dis }}(k) .
\end{aligned}
$$

Then, it is deduced from equation (25) that

$$
\rho(k)=\mathrm{GDB} u_{\text {dis }}(k-1) .
$$

It can be further obtained that

$$
\rho(k+1)-\rho(k)=h(-\delta \operatorname{sgn}(\rho(k))-q \rho(k)) .
$$

When the sampling time $h$ is very small, the existence and arrival conditions of the discrete-time sliding mode are as follows [28]:

$$
\begin{gathered}
{[\rho(k+1)-\rho(k)] \operatorname{sgn}(\rho(k))<0,} \\
{[\rho(k+1)+\rho(k)] \operatorname{sgn}(\rho(k))>0 .}
\end{gathered}
$$

From equation (27), it can be obtained that

$$
\begin{aligned}
& {[\rho(k+1)-\rho(k)] \operatorname{sgn}(\rho(k))} \\
& \quad=[h(-\delta \operatorname{sgn}(\rho(k))-q \rho(k))] \operatorname{sgn}(\rho(k)) \\
& \quad=-q h|\rho(k)|-\delta h|\rho(k)|<0 .
\end{aligned}
$$

When the sampling time $h$ is very small, $2-q h \gg 0$, and we have

$$
\begin{aligned}
& {[\rho(k+1)+\rho(k)] \operatorname{sgn}(\rho(k))} \\
& \quad=[-\delta h \operatorname{sgn}(\rho(k))+(2-q h) \rho(k)] \operatorname{sgn}(\rho(k)) \\
& \quad=(2-q h)|\rho(k)|-\delta h|\rho(k)|>0 .
\end{aligned}
$$

Thus, it can be seen that control law (23) satisfies the reachability of the sliding surface.

By utilizing the state estimated information $\widehat{x}(k)$ and the fault estimated information $\hat{f}(k)$, the following practical fault-tolerant tracking controller can be obtained:

$$
\begin{aligned}
u(k)= & (\mathrm{GDB})^{-1} G\left(W_{g}-(1+\theta) s(k)-D(A \widehat{x}(k)\right. \\
& +g(\widehat{x}(k))+E \widehat{f}(k)))+u_{\text {dis }}(k-1) \\
& +(\mathrm{GDB})^{-1} h(-\delta \operatorname{sgn}(\rho(k))-q \rho(k)) .
\end{aligned}
$$

Remark 3. The overall fault diagnosis and fault-tolerant control diagram is shown in Figure 1. The fault diagnosis and fault-tolerant process is described as follows:

(1) When fault occurs, calculate $L=P^{-1} R_{1}, v_{2}=Q^{-1} R_{2}$, and $v_{1}=Q^{-1} R_{3}$ using (12).

(2) Formulate $\hat{f}(k)$ using (8).

(3) On the basis of fault diagnosis result, a new 2-order discrete-time sliding mode fault-tolerant tracking control method is designed, and the controller $u(k)$ can be obtained by using (23).

(4) When fault occurs, the practical reconfigured controller in (31) can be obtained based on the estimated information.

\section{Simulation Examples}

To verify the effectiveness of the proposed algorithm, two illustrative examples are simulated based on the MATLAB/ Simulink platform in this section. By using the real-time toolbox in MATLAB, the proposed methodology can be implemented in real time.

Example 1. A numerical simulation is given in this example. The following functions are selected as the basis functions for approximating the output PDF:

$$
\varphi_{i}(y)=\left\{\begin{array}{ll}
|\sin 2 \pi y|, & y \in[0.5(i-1) ; 0.5 i] \\
0, & y \in[0.5(j-1) ; 0.5 j]
\end{array}, \quad i \neq j .\right.
$$




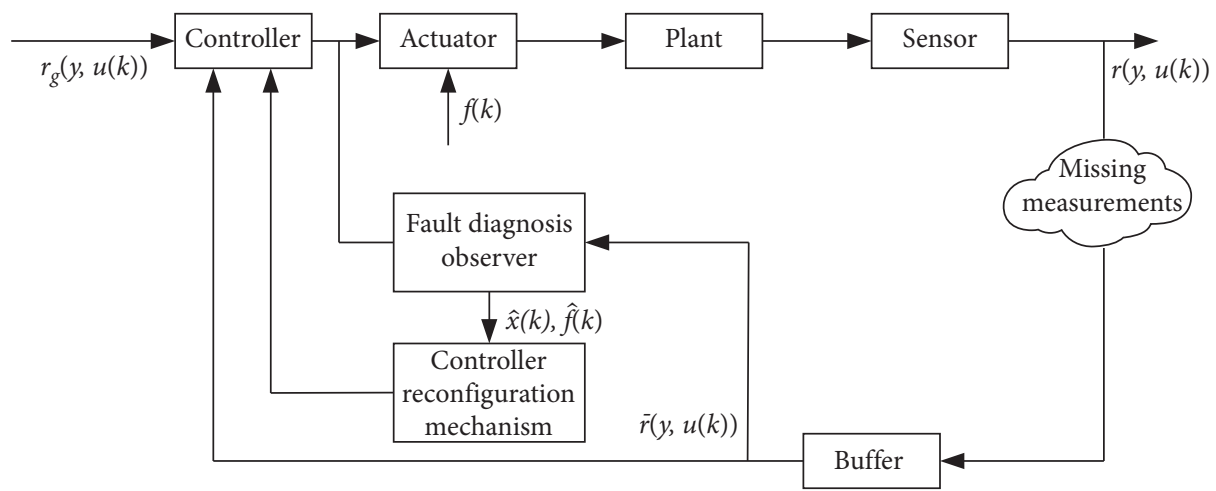

Figure 1: The overall fault diagnosis and fault-tolerant control diagram.

The system parameter matrices are given as follows:

$$
\begin{aligned}
A & =\left[\begin{array}{cc}
-1.80 & -1.21 \\
2.46 & 1.4
\end{array}\right], \\
B & =\left[\begin{array}{cc}
0.5 & -0.8 \\
-0.3 & 0.9
\end{array}\right], \\
D & =\left[\begin{array}{cc}
0.11 & 0 \\
0 & -0.2
\end{array}\right], \\
H & =\left[\begin{array}{c}
1.4 \\
1.1
\end{array}\right], \\
g(x(k)) & =\left[\begin{array}{c}
0.3\left(1-\exp ^{-x_{1}^{2}(k)}\right) \\
0.1 x_{2} \sin x_{2}
\end{array}\right], \\
W_{g} & =\left[\begin{array}{l}
0.17 \\
0.21
\end{array}\right] .
\end{aligned}
$$

By solving LMI (12), it can be obtained that

$$
\begin{aligned}
v_{1} & =1, \\
v_{2} & =0.2, \\
L & =\left[\begin{array}{c}
-0.1 \\
-0.21
\end{array}\right], \\
P & =\left[\begin{array}{ll}
0.3156 & 0.2057 \\
0.2057 & 0.1770
\end{array}\right], \\
Q & =298.9297 .
\end{aligned}
$$

It is assumed that the fault is given as follows:

$$
f(t)= \begin{cases}0, & t<30, \\ 1, & 30 \leq t \leq 60, \\ 2-\exp (-0.35(t-60)), & t>60,\end{cases}
$$

where $t=k \cdot h$.

Figure 2 shows the missing measurements when the probability of missing measurements is 0.3 in the system. The simulation results of the proposed algorithm are given in Figures 3-7. Figure 3 shows the fault estimation can track the change of fault quickly and accurately. It manifests that the fault diagnosis algorithm is effective. Figure 4 shows the input signal, and Figure 5 shows the desired output PDF. The output PDF with FTTC is shown in Figure 6, and the output

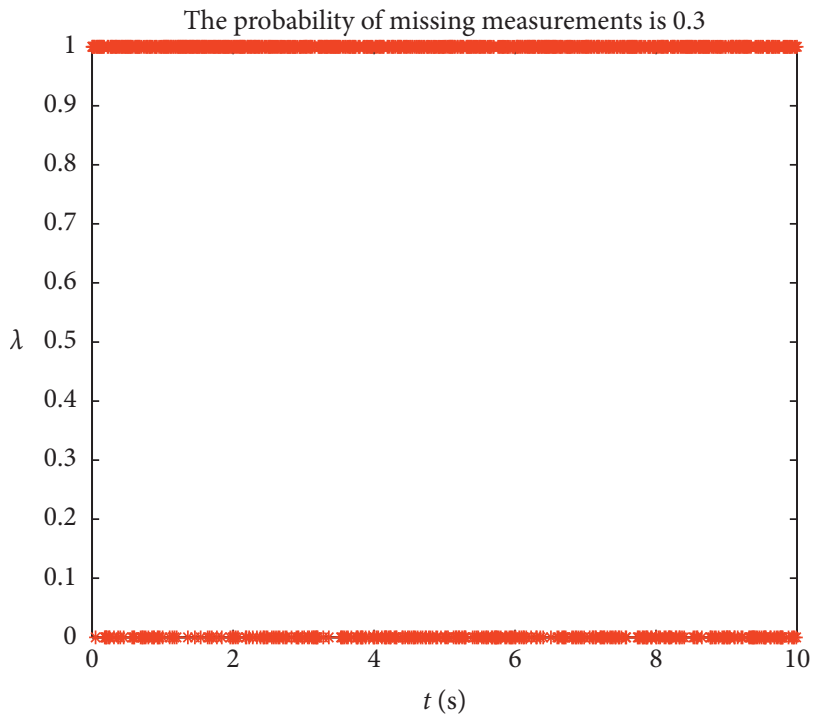

Figure 2: The missing measurements in feedback channel.

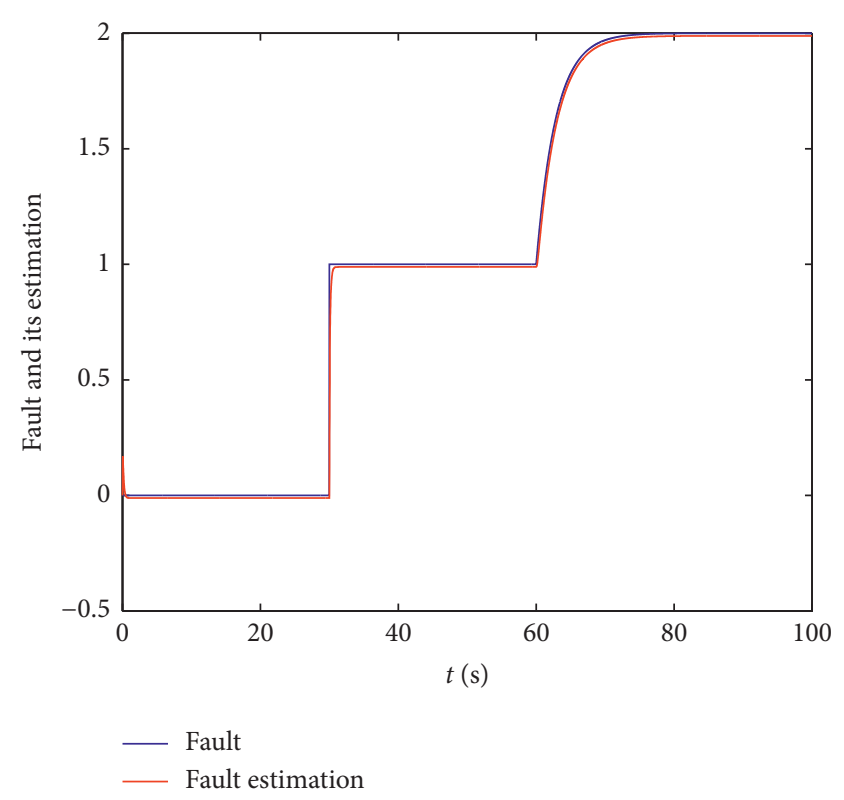

Figure 3: Fault and fault estimation. 


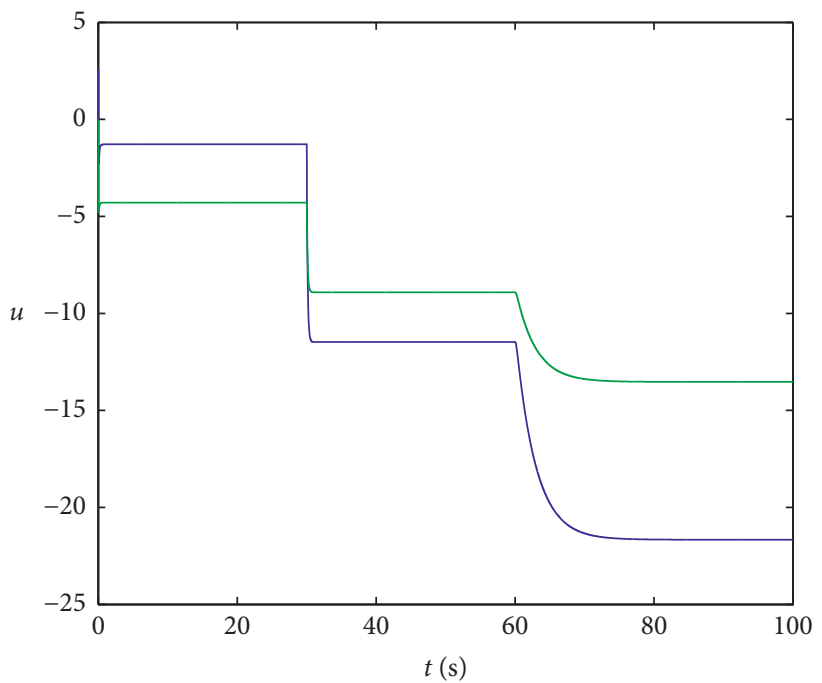

$-u 1$

$-u 2$

FIgURE 4: The input signal.

System of three-dimensional graph (PDF)

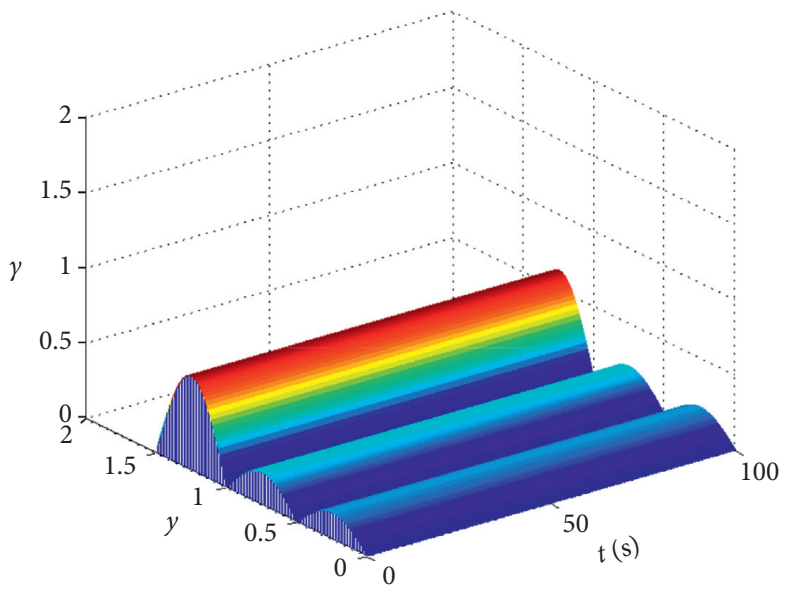

Figure 5: The desired output PDF.

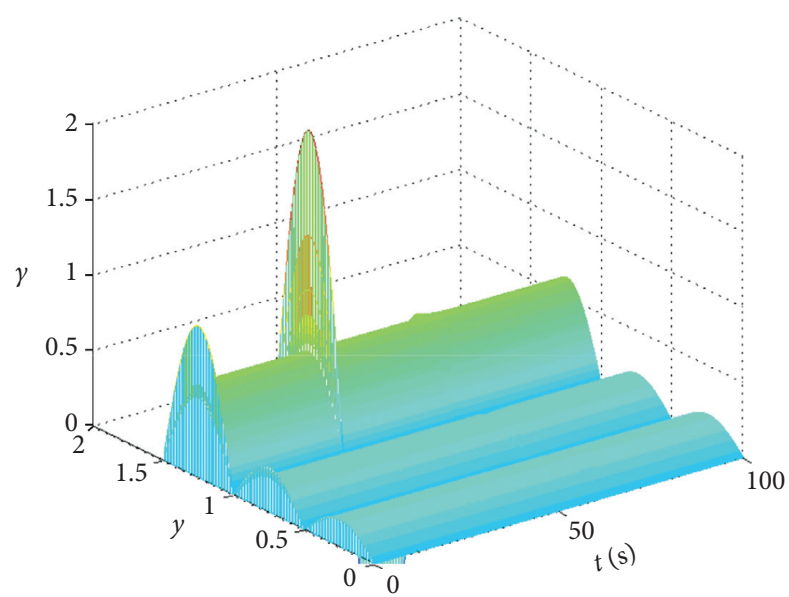

FIGURE 6: The output PDF with the proposed FTTC strategy. 
PDF presents an obvious oscillation when fault occurs at $t=30 \mathrm{~s}$, which can be returned to stability in a very short time through fault tolerance measures. The expected output PDF and the final output PDF with FTTC strategy are shown in Figure 7. It can be seen that the postfault output PDF can still follow the desired output PDF, leading to good faulttolerant tracking control results.

Example 2. In this example, a papermaking machine system [6] is considered to illustrate the applicability of the proposed method as follows:

$$
\left[\begin{array}{c}
\dot{\alpha} \\
\dot{\beta}
\end{array}\right]=\left[\begin{array}{cc}
-K_{1} & K_{2} \\
K_{3} & K_{4}-K_{2}
\end{array}\right]\left[\begin{array}{l}
\alpha \\
\beta
\end{array}\right]+\left[\begin{array}{cc}
K_{5} & 0 \\
0 & K_{6}
\end{array}\right] U(t)+\left[\begin{array}{c}
0 \\
K_{7} \sin \alpha
\end{array}\right],
$$

where $\alpha$ and $\beta$ are the shape parameter and inverse scale parameter controlling the fibre length distribution in the white water pit. The flow rate and the concentration of the retention polymer are selected as the control input $U(t)$, and $K_{1}, K_{2}, K_{3}, K_{4}$, and $K_{7}$ are machine-dependent constants which are related to the headbox geometry and the wire table arrangement. $K_{5}$ and $K_{6}$ are machine-dependent constants related to the wet design of the paper machine.

By applying the Euler discretization method, the following discrete-time system parameters can be obtained as

$$
\begin{aligned}
A & =\left[\begin{array}{cc}
-1.80 & -0.81 \\
3.44 & 1.10
\end{array}\right], \\
B & =\left[\begin{array}{cc}
-1.5 & 0 \\
0 & 1.7
\end{array}\right], \\
D & =\left[\begin{array}{cc}
0.2 & 0.1 \\
0 & 0.1
\end{array}\right], \\
H & =\left[\begin{array}{c}
0.4 \\
0.51
\end{array}\right], \\
g(x(k)) & =\left[\begin{array}{c}
0 \\
\sin x_{1}
\end{array}\right], \\
W_{g} & =\left[\begin{array}{l}
0.22 \\
0.70
\end{array}\right] .
\end{aligned}
$$

In order to approximate the output PDF, the following $\mathrm{B}$-spline functions are considered as

$$
\begin{aligned}
& \varphi_{1}(y)=\frac{1}{2}(y-2)^{2} I_{1}+\left(-y^{2}+7 y-11.5\right) I_{2}+\frac{1}{2}(y-5)^{2} I_{3}, \\
& \varphi_{2}(y)=\frac{1}{2}(y-3)^{2} I_{1}+\left(-y^{2}+9 y-19.5\right) I_{2}+\frac{1}{2}(y-6)^{2} I_{3}, \\
& \varphi_{3}(y)=\frac{1}{2}(y-4)^{2} I_{1}+\left(-y^{2}+11 y-29.5\right) I_{2}+\frac{1}{2}(y-7)^{2} I_{3},
\end{aligned}
$$

where $I_{i}(y)=\left\{\begin{array}{ll}1, & y \in[i+1, i+2] \\ 0, & \text { otherwise }\end{array}, \quad i=1,2,3,4,5\right.$.

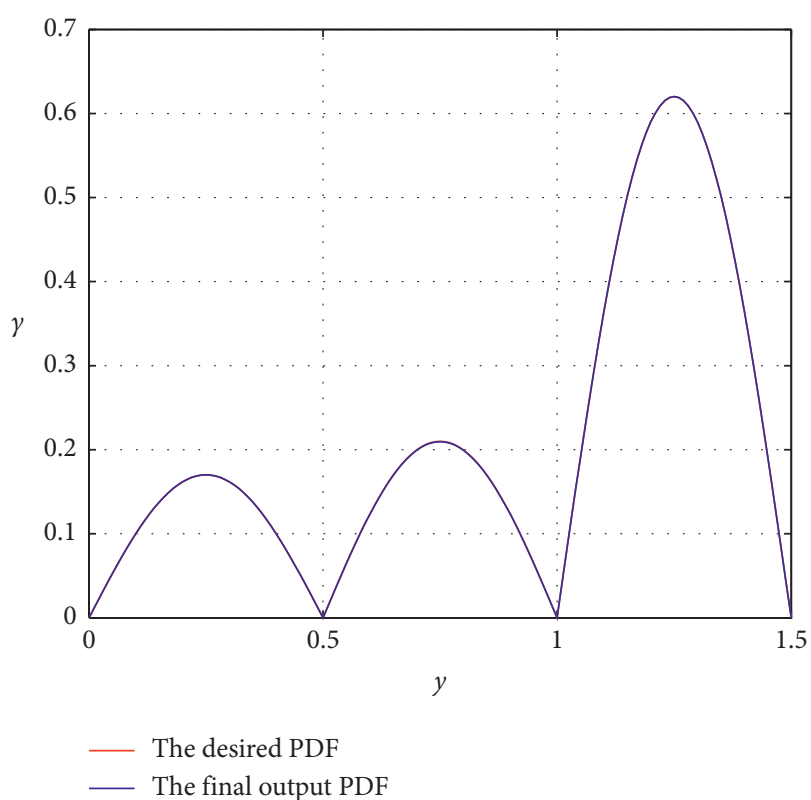

Figure 7: The expected output PDF and the final output PDF with the proposed FTTC strategy.

By solving the conditions in Theorem 1, it can be formulated as follows:

$$
\begin{aligned}
v_{1} & =1, \\
v_{2} & =-0.8, \\
L & =\left[\begin{array}{l}
-0.01 \\
-0.005
\end{array}\right], \\
P & =\left[\begin{array}{ll}
1.2710 & 0.5339 \\
0.5339 & 0.3088
\end{array}\right], \\
Q & =827.0393 .
\end{aligned}
$$

It is assumed that the form of the fault is constructed as follows:

$$
f(t)= \begin{cases}0, & t<50 \\ \sin (0.5 t), & t \geq 50 .\end{cases}
$$

When the probability of the missing measurements is 0.4 , Figure 8 shows the simulation result of the measurement signal dropouts. Figure 9 gives the results of fault diagnosis; it can be seen that the proposed fault diagnosis method has a faster action to track the changes of the fault. Figure 10 shows the input signal, and Figure 11 shows the desired output PDF. The output PDF with FTTC is shown in Figure 12. It can be seen that the degradation of the tracking performance can be eliminated with fault-tolerant measures. The expected output PDF and the final output PDF with the FTTC strategy are shown in Figure 13.

Considering the first-order sliding mode control and choosing sliding mode function $\rho(k)=G e_{w}(k)$, the corresponding fault-tolerant controller is obtained as follows: 


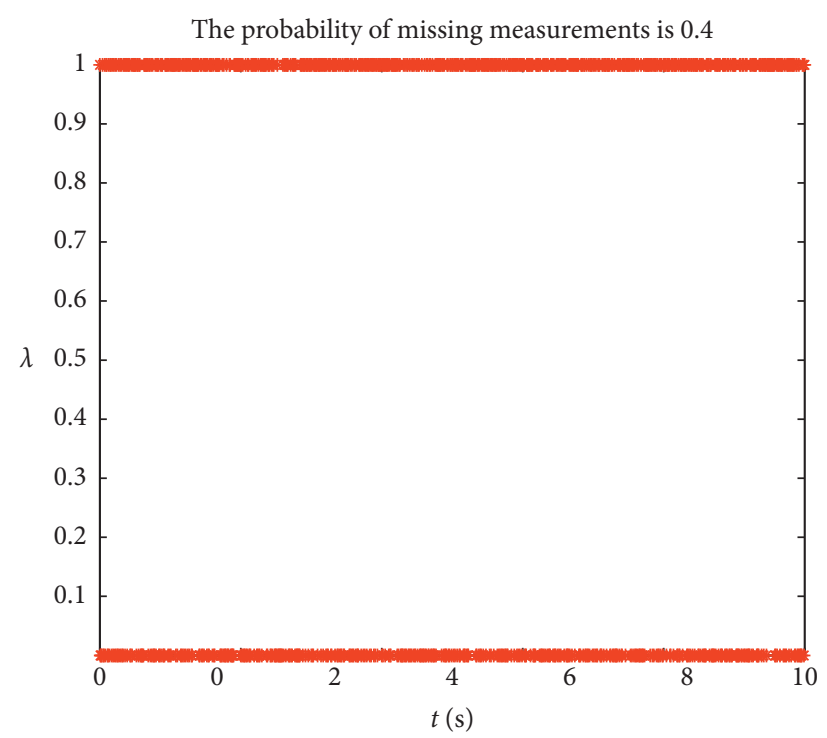

FIgURE 8: The missing measurements in feedback channel.

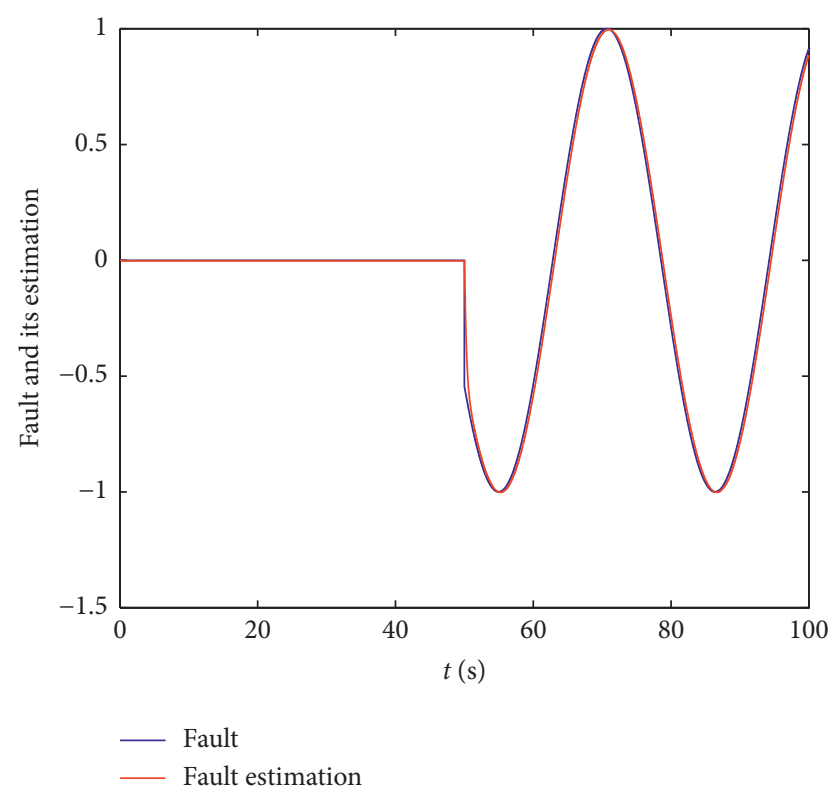

FIGURE 9: Fault and fault estimation.

$$
\begin{aligned}
u(k)= & (\mathrm{GDB})^{-1} G\left(W_{g}-D(A \hat{x}(k)+g(\widehat{x}(k))+E \hat{f}(k))\right) \\
& +u_{\mathrm{dis}}(k-1)+(\mathrm{GDB})^{-1} h(-\delta \operatorname{sgn}(\rho(k))-q \rho(k)) .
\end{aligned}
$$

Figure 14 shows the final output PDF with the first-order sliding mode fault-tolerant controller in Example 1, and Figure 15 shows the final output PDF with the first-order sliding mode fault-tolerant controller in Example 2. It can be seen that the proposed fault-tolerant controller in this paper has better tracking performance than the first-order sliding mode fault-tolerant controller.

Remark 4. From the simulation results, it can be seen that both the slow-varying fault and the fast-varying fault can be well estimated by the proposed fault diagnosis method. 


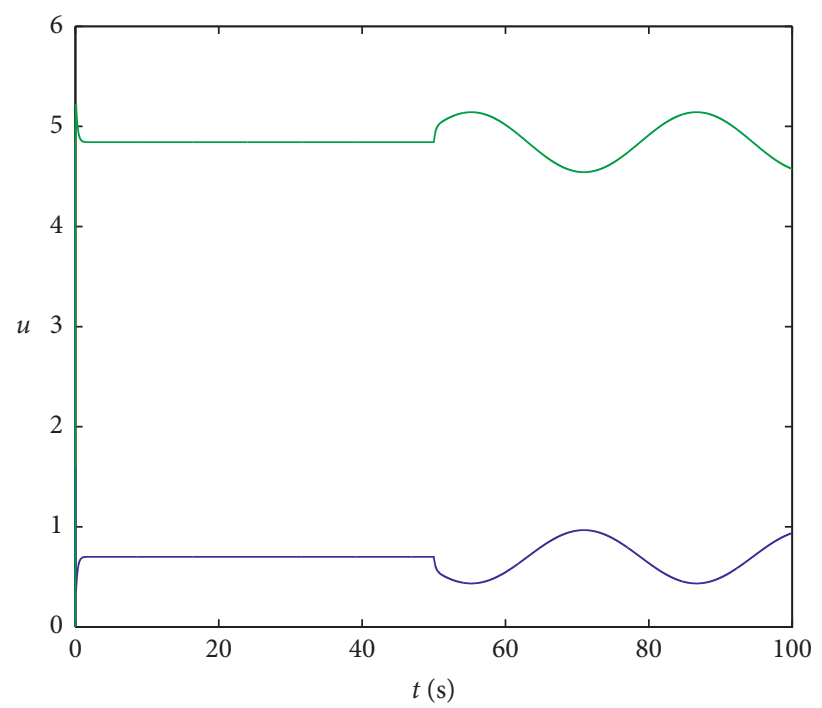

$-u 1$

$-u 2$

FIgURE 10: The input signal.

System of three-dimensional graph (PDF)

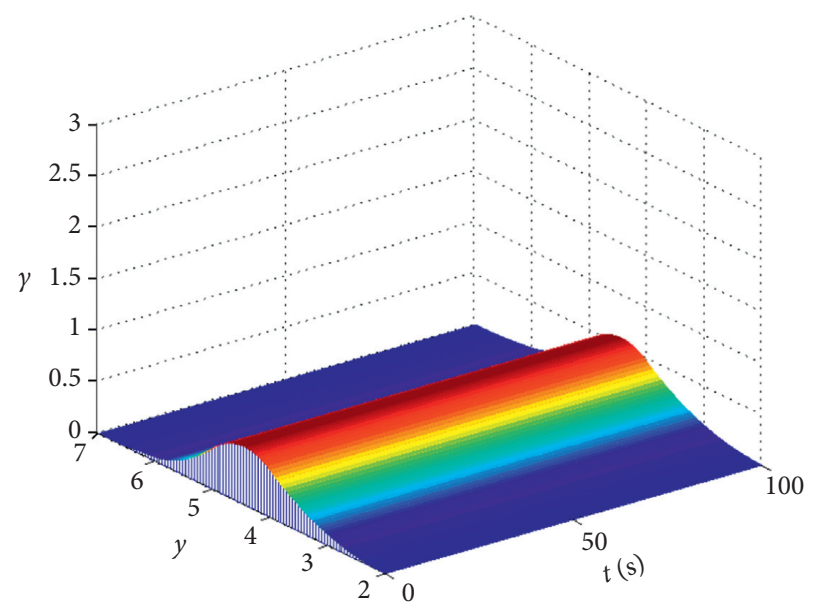

FIgURE 11: The desired output PDF.

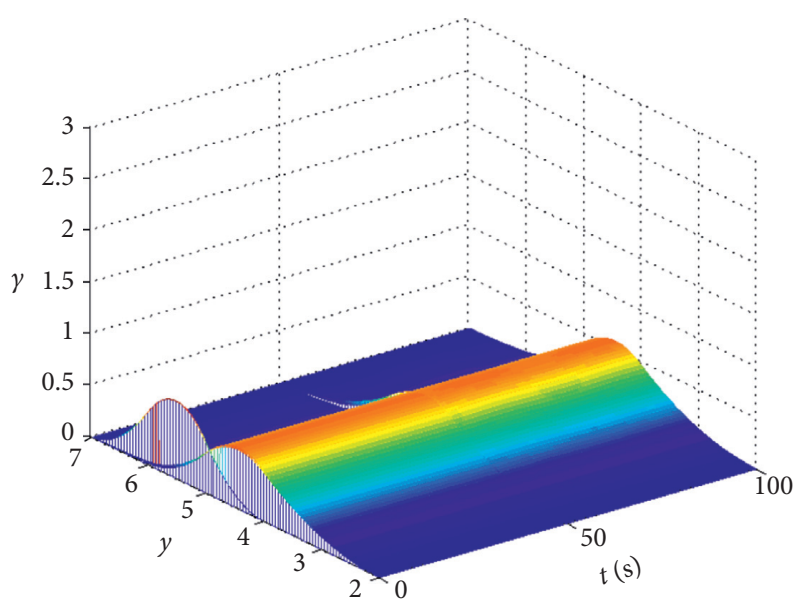

FIgURE 12: The output PDF with the proposed FTTC strategy. 


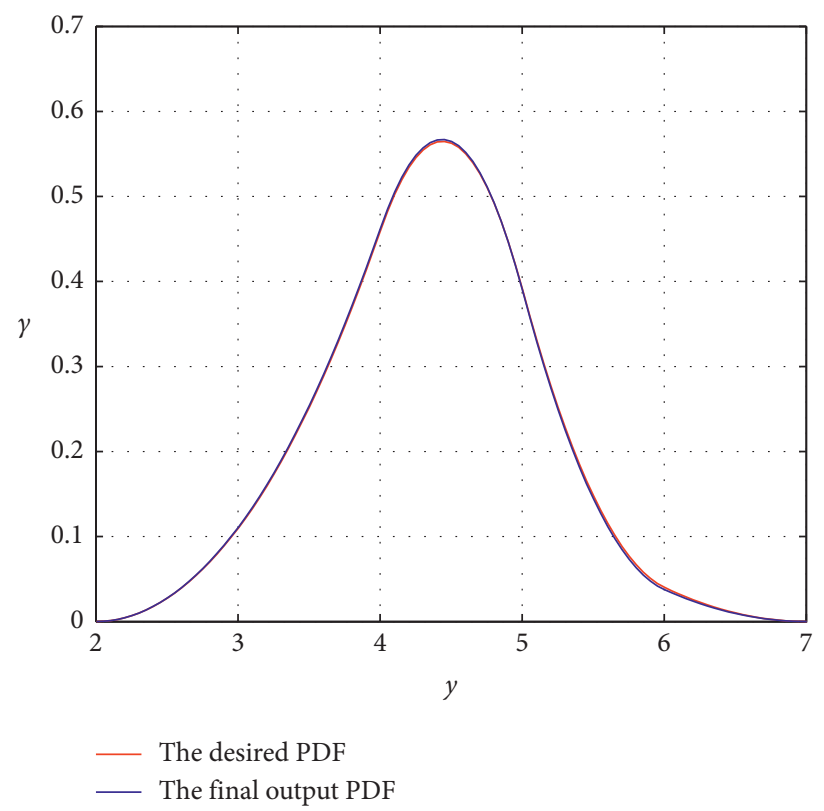

Figure 13: The expected output PDF and the final output PDF with the proposed FTTC strategy.

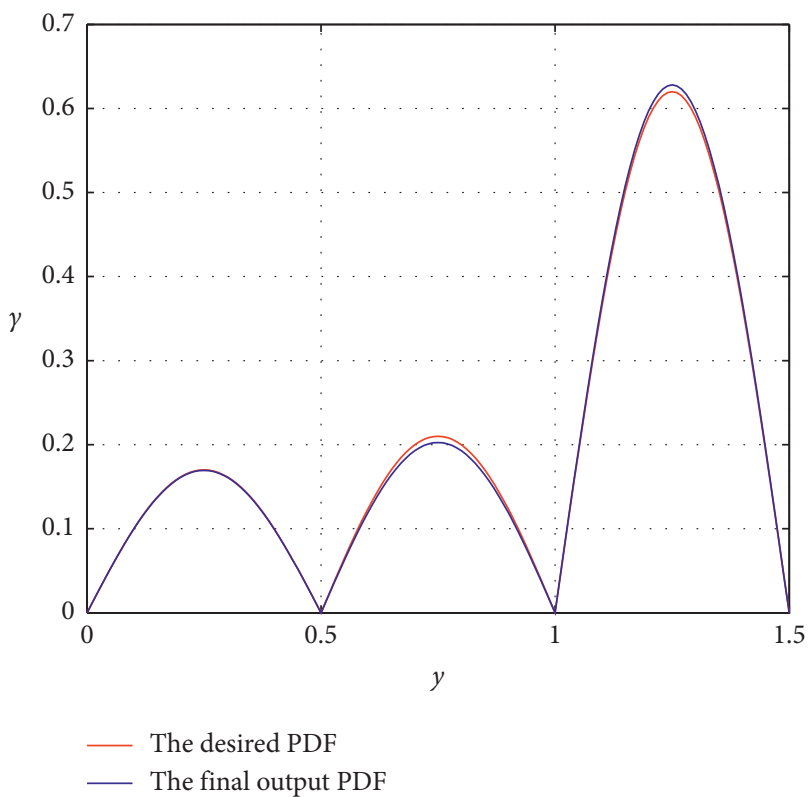

FIgURE 14: The expected output PDF and the final output PDF with the first-order sliding mode FTTC strategy.

Remark 5. In this paper, we just focus on the fault diagnosis and fault-tolerant control for the actuator additive fault. The problem of fault-tolerant control of actuator multiplicative fault and sensor fault for stochastic distribution control systems with missing measurements is still a challenging one, which will be our future research work.

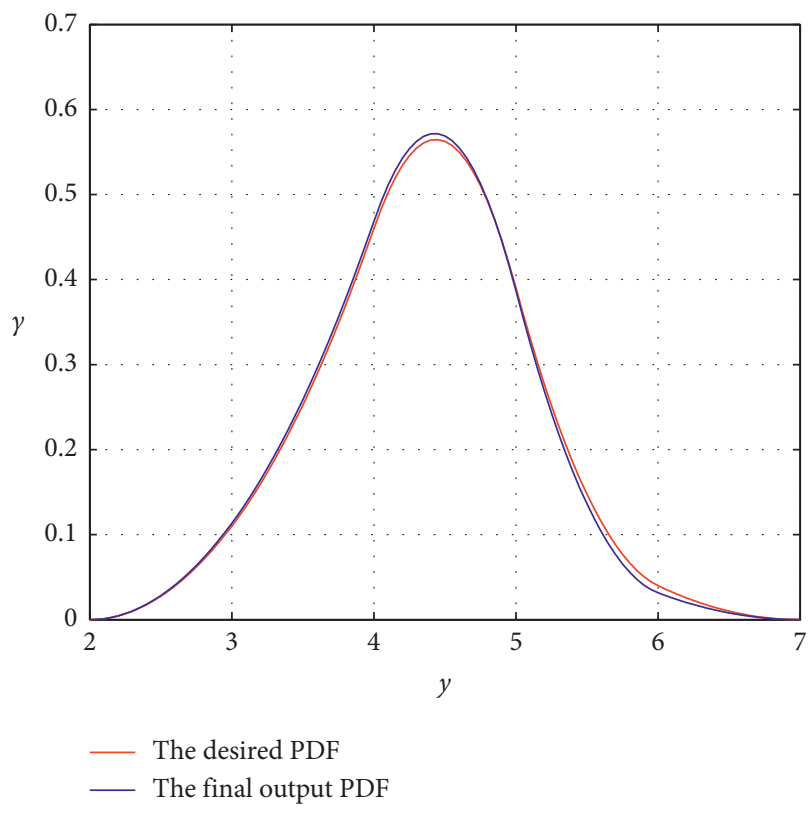

FIGURE 15: The expected output PDF and the final output PDF with the first-order sliding mode FTTC strategy.

\section{Conclusions}

In this paper, the fault-tolerant tracking control problem is investigated for non-Gaussian nonlinear SDC systems with missing measurements. The phenomenon of missing measurements is described by a random variable satisfying the Bernoulli distribution. An adaptive fault diagnosis observer 
is designed to estimate the state and fault. Then, a new discrete-time 2-order sliding mode fault-tolerant tracking controller is designed to make the postfault output PDF still track the desired one. At the same time, it can be proved that the control law can guarantee the reachability of the sliding surface. The simulation results show that the proposed algorithm not only can tolerate high data packet dropout rate but also has good fault-tolerant tracking results.

\section{Data Availability}

All data, models, and code generated or used during the study are included within the article.

\section{Conflicts of Interest}

The authors declare that they have no conflicts of interest.

\section{Acknowledgments}

This study was supported by the NSFC grants (nos. 61973278 and 61374128).

\section{References}

[1] M. Basin, P. Shi, and D. Calderon-Alvarez, "Joint state filtering and parameter estimation for linear stochastic timedelay systems," Signal Processing, vol. 91, no. 4, pp. 782-792, 2011.

[2] T. Damm, "Generalized riccati equations and stabilization of stochastic systems," IFAC Proceedings Volumes, vol. 33, no. 16, pp. 437-442, 2000.

[3] P. Ni, J. Li, H. Hao, and Y. Xia, "Stochastic dynamic analysis of marine risers considering Gaussian system uncertainties," Journal of Sound and Vibration, vol. 416, no. 3, pp. 224-243, 2018.

[4] H. Wang, Bounded Dynamic Stochastic Systems: Modeling and Control, Springer-Verlag, London, UK, 2000.

[5] T. Li and L. Guo, "Optimal fault-detection filtering for nonGaussian systems via output PDFs," IEEE Transactions on Systems, Man, and Cybernetics Part A:Systems and Humans, vol. 39, no. 2, pp. 476-481, 2009.

[6] Y. Yi, Y.-Y. Zhao, and S.-Y. Cao, "Fuzzy filter-based FDD design for non-Gaussian stochastic distribution processes using T-S Fuzzy modeling," Mathematical Problems in Engineering, vol. 2013, Article ID 156262, 7 pages, 2013.

[7] J. Zhou, H. Yue, J. Zhang, and H. Wang, "Iterative learning double closed-loop structure for modeling and controller design of output stochastic distribution control systems," IEEE Transactions on Control Systems Technology, vol. 22, no. 6, pp. 2261-2276, 2014.

[8] L. Yin and L. Guo, "Joint stochastic distribution tracking control for multivariate descriptor systems with nonGaussian variables," International Journal of Systems Science, vol. 43, no. 1, pp. 192-200, 2012.

[9] J. Zhou, G. Li, and H. Wang, "Robust tracking controller design for non-Gaussian singular uncertainty stochastic distribution systems," Automatica, vol. 50, no. 4, pp. 12961303, 2014.

[10] Y. Wu and J. Dong, "Tracking control for non-Gaussian stochastic distribution sampled-data fuzzy systems," Fuzzy Sets and Systems, vol. 356, no. 1, pp. 1-27, 2019.
[11] H. Yue, H. Wang, and J. Zhang, "Shaping of molecular weight distribution by iterative learning probability density function control strategies," Proceedings of the Institution of $\mathrm{Me}$ chanical Engineers, Part I: Journal of Systems and Control Engineering, vol. 222, no. 7, pp. 639-653, 2008.

[12] J. Zhang, H. Yue, and J. Zhou, "Predictive PDF control in shaping of molecular weight distribution based on a new modeling algorithm," Journal of Process Control, vol. 30, no. 1, pp. 80-89, 2015.

[13] P. Kabore, H. Baki, H. Yue, and H. Wang, "Linearized controller design for the output probability density functions of non-Gaussian stochastic systems," International Journal of Automation and Computing, vol. 2, no. 1, pp. 67-74, 2005.

[14] Z. Skaf, H. Wang, and L. Guo, "Fault tolerant control based on stochastic distribution via RBF neural networks," Journal of Systems Engineering and Electronics, vol. 22, no. 1, pp. 63-69, 2011.

[15] L. Yao, J. Qin, H. Wang, and B. Jiang, "Design of new fault diagnosis and fault tolerant control scheme for non-Gaussian singular stochastic distribution systems," Automatica, vol. 48, no. 9, pp. 2305-2313, 2012.

[16] Y. Sun and L. Yao, "Robust fault diagnosis and fault-tolerant control for non-Gaussian uncertain stochastic distribution control systems," International Journal of Robust and Nonlinear Control, vol. 27, no. 10, pp. 1709-1725, 2017.

[17] G. Li and Q. Zhao, "Adaptive fault-tolerant shape control for nonlinear Lipschitz stochastic distribution systems," Journal of the Franklin Institute, vol. 354, no. 1, pp. 4013-4033, 2017.

[18] Y. Ren, Y. Fang, A. Wang, H. Zhang, and H. Wang, "Collaborative operational fault tolerant control for stochastic distribution control system," Automatica, vol. 98, no. 1, pp. 141-149, 2018.

[19] J. M. Chen, "System parameter estimation with input/output noisy data and missing measurements," IEEE Transactions on Signal Processing, vol. 48, no. 6, pp. 1548-1558, 2000.

[20] L. Hu, W. Z. Wang, I. Rahman, and X. H. Liu, "A constrained optimization approach to dynamic state estimation for power systems including PMU and missing measurements," IEEE Transactions on Control Systems Technology, vol. 24, no. 2, pp. 703-710, 2016.

[21] S. Wang, X. Tian, and H. Fang, "Event-based state and fault estimation for nonlinear systems with logarithmic quantization and missing measurements," Journal of the Franklin Institute, vol. 356, no. 7, pp. 4076-4096, 2019.

[22] M. Nasiri, S. Mobayen, and Q. M. Zhu, "Super-twisting sliding mode control for gearless PMSG-based wind turbine," Complexity, vol. 2019, Article ID 6141607, 15 pages, 2019.

[23] H. B. Wang, B. Su, Y. L. Wang, and J. Gao, "Adaptive sliding mode fixed-time tracking control based on fixed-time sliding mode disturbance observer with dead-zone input," Complexity, vol. 2019, Article ID 8951382, 14 pages, 2019.

[24] S. M. Rakhtala, M. Yasoubi, and H. HosseinNia, "Design of second order sliding mode and sliding mode algorithms: a practical insight to DC-DC buck converter," IEEE/CAA Journal of Automatica Sinica, vol. 4, no. 3, pp. 483-497, 2017.

[25] Y. A. Alma, A. D. Nancy, and L. F. Carlos, "Bacterial foraging optimization algorithm to improve a discrete-time neural second order sliding mode controller," Applied Mathematics and Computation, vol. 271, no. 1, pp. 43-51, 2015.

[26] H. Romdhane, K. Dehri, and A. S. Nouri, "Discrete secondorder sliding mode control based on optimal sliding function vector for multivariable systems with input-output representation," International Journal of Robust and Nonlinear Control, vol. 26, no. 17, pp. 3806-3830, 2016. 
[27] M. Li, P. Zhou, H. Wang, and T. Chai, "Geometric analysis based double closed-loop iterative learning control of output PDF shaping of fiber length distribution in refining process," IEEE Transactions on Industrial Electronics, vol. 66, no. 9, pp. 7229-7238, 2019.

[28] S. Sarpturk, Y. Istefanopulos, and O. Kaynak, "On the stability of discrete-time sliding mode control systems," IEEE Transactions on Automatic Control, vol. 32, no. 10, pp. 930-932, 1987. 